\title{
APPROXIMATE $\ell$-STATE SOLUTION OF TIME INDEPENDENT SCHRÖDINGER WAVE EQUATION WITH MODIFIED MÖBIUS SQUARED POTENTIAL PLUS HULTHÉN POTENTIAL
}

\author{
${ }^{* 1}$ Yabwa, D., ${ }^{2}$ Eyube, E. S. and ${ }^{3}$ Ibrahim, Y. \\ ${ }^{1}$ Department of Physics, Faculty of Science, Taraba State University, Jalingo, Nigeria \\ ${ }^{2}$ Department of Physics, School of Physical Sciences, Modibbo Adama University of Technology, Yola, Nigeria \\ ${ }^{3}$ Department of Science Laboratory Technology, Gombe State Polytechnic Bajoga, Nigeria \\ *Corresponding Author's Email: yabwa.dlama@tsuniversity.edu.ng Tel: +2348133275536
}

\begin{abstract}
In this work we have applied ansatz method to solve for the approximate $\ell$-state solution of time independent Schrödinger wave equation with modified Möbius squared potential plus Hulthén potential to obtain closed form expressions for the energy eigenvalues and normalized radial wave-functions. In dealing with the spinorbit coupling potential of the effective potential energy function, we have employed the Pekeris type approximation scheme, using our expressions for the bound state energy eigenvalues, we have deduced closed form expressions for the bound states energy eigenvalues and normalized radial wave-functions for Hulthén potential, modified Möbius square potential and Deng-Fan potential. Using the value 0.976865485225 for the parameter $\omega$, we have computed bound state energy eigenvalues for various quantum states (in atomic units). We have also computed bound state energy eigenvalues for six diatomic molecules: $\mathrm{HCl}, \mathrm{LiH}, \mathrm{TiH}, \mathrm{NiC}, \mathrm{TiC}$ and $\mathrm{ScF}$. The results we obtained are in near perfect agreement with numerical results in the literature and a clear demonstration of the superiority of the Pekeris-type approximation scheme over the Greene and Aldrich approximation scheme for the modified Möbius squares potential plus Hulthén potential.
\end{abstract}

Keywords: Ansatz solution, Deng-Fan potential, energy eigenvalues, radial wavefunction, Pekeris approximation schemes

\section{INTRODUCTION}

The need for exact solution of Schrödinger wave equation in quantum mechanics cannot be over emphasized, this is due to the vital information derivable from them (Miranda et al., 2010; Qiang, et al., 2009), information such as energy, momentum, wavelength and frequency of the system can only be obtained with the knowledge of the wave function (Eyube et al., 2019). Exact solution of the Schrödinger equation is restricted to only few potential models such as the Coulombic potential and harmonic oscillator potential (Hitler et al., 2017; Tsaur and Wang, 2014) for all quantum states $n \ell$ where $n$ is the principal quantum number and $\ell$ is the angular momentum quantum number. The Hulthén, Morse, and Eckart potentials are among the few potential energy functions which give exact solution for zero angular momentum quantum number $(\ell=0)$, these solutions are often referred to as s-wave solutions (Hitler et al., 2017; Tsaur and Wang, 2014). Most of the known potential energy functions have no exact solutions with the Schrödinger equation for all values of $n$ and $\ell$, for such potentials, approximate solutions (numerical or analytical) can be used in place of exact solutions (Rahbar and Sadeghi,2016). In order to obtain approximate analytical solution, a very suitable approximation scheme (Wei and Dong, 2010; Chen et al., 2009; Jia et al, 2008) must be applied on the spin-orbit term of the effective potential, having applied the approximation model on the centrifugal term, a solution method must be adopted to solve the resulting equation. Researchers have developed and used various solution methods to solve the Schrödinger equation, amongst some of the methods include: ansatz method (Taskin and Kocal, 2010), Nikiforov-Uvarov method (Ikot et al., 2014; Yazarloo et al., 2012), factorization method (Pahlavani et al., 2013), asymptotic iteration method (Awoga and Ikot 2012), Fröbenius series solution method (Nyengeri et al., 2018), exact quantization rule (Qiang et al 2008). Various forms of potential energy functions have been used to solve the radial Schrödinger equation. Yazarloo et al. (2012) have obtained the oscillator strength for the modified Möbius square potential. The Schrödinger equation was studied by an improved approximation scheme for the Hulthén potential (Ikhdair,2009), Okorie et al. (2018) have studied the solution of the Schrödinger equation with modified Möbius square potential, they used their results to explore the thermodynamic properties of the potential. Researchers have also used combined potentials to the radial Schrödinger equation. Recently Ita et al. (2018) have solved the Schrödinger equation with the Woods-Saxon plus attractive inversely quadratic potential for the bound state solution Hitler et al. (2017) have applied the Nikiforov-Uvarov method to solve for the s-wave solution of Schrodinger equation with Kratzer plus modified Deng-Fan potential, in another event Edet and collaborators have used modified Kratzer potential plus screened coulomb potential to study the Schrödinger equation (Edet et al., 2019). The list of authors who have used combined potentials seems to endless in the literature. In this paper, we are encouraged to solve for the approximate $\ell$-state solution of time independent Schrödinger wave equation with modified Möbius squared potential plus Hulthén potential, which, to the best of our knowledge has never been solved in the literature. Our specific task is to obtain (i) closed form expressions for the bound state energy eigenvalues and normalized radial wave functions of the modified Möbius square potential plus Hulthén potential, (ii) use our results to derive expressions for the bound state energy eigenvalues and radial wave functions of Hulthén potential, modified Möbius square potential and Deng-Fan potential, (iii) use our results to compute bound state energy eigenvalues of Hulthén potential and Deng-fan potential for arbitrary values of quantum numbers, (iv) compare our results with existing results in the literature. 


\section{Theoretical Formalism}

The modified Möbius squared potential plus Hulthén potential

$$
V(r)=-V_{0}\left(\frac{A+B e^{-2 \delta r}}{1-e^{-2 \delta r}}\right)^{2}-\frac{Z e^{2} \delta e^{-\delta r}}{1-e^{-\delta r}}
$$

where $V_{0}, A, B$ and $\delta$ are the depth of the potential, the range of the potential, the length of the molecular bond and the adjustable screening parameter, $Z$ is a constant which is related to the atomic number of an element. $V(r)$ is the modified Mobius square potential plus the Hulthén potential. The effective potential is given by

$$
V_{e f f}(r)=-V_{0}\left(\frac{A+B e^{-2 \delta r}}{1-e^{-2 \delta r}}\right)^{2}+\frac{Z e^{2} \delta e^{-\delta r}}{1-e^{-\delta r}}+\frac{L \hbar^{2}}{2 \mu r^{2}} .
$$

$L=\ell(\ell+1), \mu$ is the mass of the system, $r$ is the internuclear separation and $\hbar$ is the reduced Planck's constant.

The radial Schrödinger equation

The radial Schrödinger equation reads (Okorie et al.,2018)

$$
\frac{d^{2} \psi_{n l}}{d r^{2}}+\frac{2 \mu}{\hbar^{2}}\left[E_{n l}-V_{e f f}(r)\right] \psi_{n l}(r)=0
$$

where $E_{n}$ is the energy spectrum of the potential, $\psi_{n l}(r)$ is the radial wavefunction. Substituting Eq. (2) into Eq. (3) gives

$$
\frac{d^{2} \psi_{n l}}{d r^{2}}+\frac{2 \mu}{\hbar^{2}}\left[E_{n l}+V_{0}\left(\frac{\mathrm{A}+\mathrm{B} e^{-\delta r}}{1-e^{-\delta r}}\right)^{2}+\frac{Z e^{2} \delta e^{-\delta r}}{1-e^{-\delta r}}-\frac{L \hbar^{2}}{2 \mu r^{2}}\right] \psi_{n l}(r)=0
$$

Eq. (4) have exact solution for the special case of the s-wave $(\ell=0)$. However, if $\ell \neq 0$ only approximate solutions can be obtained due to the presence of the centrifugal term potential (spin-orbit coupling term). By employing a suitable approximation scheme to deal with the centrifugal term. In this article we will use Pekeris type approximation model proposed by Jia et al. (Jia et al 2008) given below:

$$
\frac{1}{r^{2}} \approx \delta^{2}\left\{\frac{\omega e^{-\delta r}}{1-e^{-\delta r}}+\frac{e^{-2 \delta r}}{\left(1-e^{-\delta r}\right)^{2}}\right\}
$$

where $\omega$ is an adjustable dimensionless parameter. Inserting the approximation expression Eq. (5) into Eq. (4) now turns to:

$$
\frac{d^{2} \psi_{n l}}{d r^{2}}+\frac{2 \mu}{\hbar^{2}}\left\{E_{n l}+V_{0}\left(\frac{\mathrm{A}+\mathrm{B} e^{-\delta r}}{1-e^{-\delta r}}\right)^{2}+\frac{Z e^{2} \delta e^{-\delta r}}{1-e^{-\delta r}}-\frac{L \hbar^{2} \delta^{2}}{2 \mu}\left[\frac{\omega e^{-\delta r}}{1-e^{-\delta r}}+\frac{e^{-2 \delta r}}{\left(1-e^{-\delta r}\right)^{2}}\right]\right\} \psi_{n l}(r)=0
$$

with the approximation given by Eq. (5) substituted in Eq. (2) gives:

$$
V_{e f f}(r)=-V_{0}\left(\frac{\mathrm{A}+\mathrm{B} e^{-\delta r}}{1-e^{-\delta r}}\right)^{2}-\frac{Z e^{2} \delta e^{-\delta r}}{1-e^{-\delta r}}+\frac{L \hbar^{2} \delta^{2}}{2 \mu}\left[\frac{\omega e^{-\delta r}}{1-e^{-\delta r}}+\frac{e^{-2 \delta r}}{\left(1-e^{-\delta r}\right)^{2}}\right]
$$

To express Eq. (6) in a more compact form, we have used the following coordinate transformation of the form

$$
z=e^{-\delta r}
$$

Eq. (6) reduces to

$$
\frac{d^{2} \psi_{n l}(r)}{d r^{2}}+\frac{2 \mu}{\hbar^{2}}+\left[E_{n l}+\frac{Z e^{2} \delta z}{(1-z)}+V_{0}\left(\frac{A+B z}{(1-z)}\right)^{2}-\frac{L \hbar^{2} \delta^{2}}{2 \mu}\left(\frac{\omega z}{1-z}+\frac{z^{2}}{(1-z)^{2}}\right)\right] \psi_{n l}(z)=0
$$

Further reduction, results to,

$$
z^{2} \frac{d^{2} \psi_{n l}(z)}{d z^{2}}+z \frac{d \psi_{n l}(z)}{d z}+\left[-e_{1}+\frac{e_{2}}{(1-z)}-\frac{2 L z+e_{3}-L}{(1-z)^{2}}\right] \psi_{n l}(z)
$$

where 


$$
\begin{aligned}
& -e_{1}=\frac{2 \mu}{\delta^{2} \hbar^{2}}\left(E_{n l}-Z e^{2} \delta+V_{0} B^{2}\right)-L(1-\omega) \\
& -e_{2}=\frac{4 \mu V_{0} B(A+B)}{\delta^{2} \hbar^{2}}-\frac{2 \mu Z e^{2} \delta}{\delta^{2} \hbar^{2}}+L \omega \\
& -e_{3}=\frac{2 \mu V_{0}(A+B)^{2}}{\hbar^{2} \delta^{2}} .
\end{aligned}
$$

The boundary condition on the wave function $\psi_{n l}(z)$ is as: $r \rightarrow 0, z \rightarrow 1$ and as $r \rightarrow \infty, z \rightarrow 0$. Under this condition, for bound state solutions we can choose a reasonable physical wavefunction as follows (Dong and Gu, 2007):

$$
\psi_{n l}(z)=N_{n l} z^{a}(1-z)^{b} f_{n l}(z) .
$$

where $a$ and $b$ are constants to be satisfied by Eq. (9) and $N_{n e}$ is the normalization constant to be determined by normalization condition. Substituting the wavefunction given by Eq. (14) into Eq. (9), we obtained

$$
z(1-z) \frac{d^{2} f_{n l}(z)}{d z^{2}}+[2 a+1-(2 a+2 b+1) z] \frac{d f_{n l}(z)}{d z}-\left[\left((a+b)^{2}-e_{1}\right)+\frac{b^{2}-b-L-e_{3}}{1-z}+\frac{a^{2}-e_{1}+e_{2}-e_{3}+L}{z}\right] f_{n l}(z)=0 .
$$

Eq. (15) is Gaussian hypergeometric (Dong and $\mathrm{Gu}, 2007$ ) if and only if

$$
a=\sqrt{e_{1}-e_{2}+e_{3}-L}
$$

and

$$
b=\frac{1}{2}+\sqrt{\frac{1}{4}+L+e_{3}}
$$

which reduces Eq. (15) into the following form

$$
z(1-z) \frac{d^{2} f_{n l}(z)}{d z^{2}}+[2 a+1-(2 a+2 b+1) z] \frac{d f_{n l}(z)}{d z}-\left[(a+b)^{2}-e_{1}\right] f_{n l}(z)=0
$$

Eq. (18) has solution given by (Dong and Wei, 2008):

$$
f_{n l}(z)={ }_{2} F_{1}(\alpha, \beta, \gamma ; z)
$$

where $f_{n l}(z)$ as given by Eq. (19) is the hypergeometric function and:

$$
\begin{aligned}
& \alpha=a+b+\sqrt{e_{1}} . \\
& \beta=a+b-\sqrt{e_{1}} \\
& \gamma=2 a+1 .
\end{aligned}
$$

For a polynomial solution, the quantum condition (Jia et al., 2008) must be satisfied, this requires that either $\alpha$ or $\beta$ must be an integer, that is

$$
\beta=-n
$$

using Eq. (11) in Eqs. (21) and (23) we obtained

$$
n+b+\sqrt{e_{1}-e_{2}+e_{3}-L}=\sqrt{e_{1}} .
$$

and

$$
E_{n l}=-\frac{\delta^{2} \hbar^{2}}{2 \mu}\left\{\frac{e_{2}-e_{3}+L}{2(n+b)}-\frac{n+b}{2}\right\}^{2}+Z e^{2} \delta-V_{0} B^{2}-\frac{\delta^{2} \hbar^{2}}{2 \mu}\left(e_{2}-e_{3}+L\right) .
$$

Eq. (25) transforms to: 


$$
\begin{array}{r}
E_{n l}=-\frac{\delta^{2} \hbar^{2}}{2 \mu}\left\{\frac{\frac{2 \mu V_{0}\left(A^{2}-B^{2}\right)}{\delta^{2} \hbar^{2}}+\frac{2 \mu Z e^{2}}{\delta \hbar^{2}}-\ell(\ell+1)(1-\omega)}{2 n+1+\sqrt{(2 \ell+1)^{2}-\frac{8 \mu V_{0}(A+B)^{2}}{\delta^{2} \hbar^{2}}}}-\frac{2 n+1+\sqrt{(2 \ell+1)^{2}-\frac{8 \mu V_{0}(A+B)^{2}}{\delta^{2} \hbar^{2}}}}{4}\right\} \\
+Z e^{2} \delta-V_{0} B^{2}-\frac{\delta^{2} \hbar^{2}}{2 \mu}\left\{\frac{2 \mu V_{0}\left(A^{2}-B^{2}\right)}{\delta^{2} \hbar^{2}}+\frac{2 \mu Z e^{2} \delta}{\delta^{2} \hbar^{2}}-\ell(\ell+1)(1-\omega)\right\}
\end{array}
$$

Eq. (26) is the expression for the bound state energy eigenvalues of the modified Möbius square potential plus Hulthén potential.

\section{Normalization constant}

The normalization of the wave function (Saxena, 2012) requires that

$$
\int_{0}^{\infty}\left|\psi_{n l}(r)\right|^{2} d r=1
$$

where $\left|\psi_{n \ell}(r)\right|^{2}=\psi_{n \ell}^{*}(r) \psi_{n \ell}(r)$ and $\psi_{n \ell}^{*}(r)$ is the complex conjugate of $\psi_{n \ell}(r)$

Eq. (27) can be expressed in terms of the variable $z$ by inserting Eqs. (8) and (14) in Eq. (27), giving rise to:

$$
\int_{0}^{1} z^{-1}\left|N_{n l} z^{a}(1-z)^{b} f_{n l}(z)\right|^{2} d z=\delta
$$

the hypergeometric function has the form, $f_{n l}(z)={ }_{2} F_{1}(\alpha, \beta, \gamma ; z)$ from Eq. (19) upon inserting the values of $\alpha, \beta$ and $\gamma$ from Eq. (20), Eq. (21) and Eq. (22) results to,

$$
f_{n l}(z)={ }_{2} F_{1}(-n, n+2 a+2 b ; 2 a+1 ; z)
$$

Putting Eq. (29) into Eq. (28), we get,

$$
\begin{aligned}
& \left.\left.N_{n l}^{2} \int_{0}^{1} z^{2 a-1}(1-z)^{2 b}\right|_{2} F_{1}(-n, n+2 a+2 b ; 2 a+1 ; z)\right|^{2} d z=\delta \\
& N_{n_{l}}=\left(\frac{\delta}{S}\right)^{\frac{1}{2}}
\end{aligned}
$$

where

$$
S=\int_{0}^{1} z^{2 a-1}(1-z)^{2 b}\left|{ }_{2} F_{1}(-n, n+2 a+2 b ; 2 a+1 ; z)\right|^{2} d z
$$

Following (Miranda et al., 2010), we find:

$$
S=\frac{n !(n+b) \Gamma(n+2 b) \Gamma(2 a) \Gamma(2 a+1)}{(n+a+b) \Gamma(n+2 a+1) \Gamma(n+2 a+2 b)}
$$

\section{RESULTS AND DISCUSSION}

\section{Hulthén potential}

If we set $A=B=O$ in Eq. (7), we have the expression for the effective Hulthén potential (Jia et al., 2008)

$$
V_{e f f}(r)=-\frac{Z e^{2} \delta e^{-\delta r}}{1-e^{-\delta r}}+\frac{\ell(\ell+1) \hbar^{2} \delta^{2}}{2 \mu}\left[\frac{\omega e^{-\delta r}}{1-e^{-\delta r}}+\frac{e^{-2 \delta r}}{\left(1-e^{-\delta r}\right)^{2}}\right]
$$

Therefore, by substituting the above values of A and B in Eq. (26), we have the energy eigenvalue for the Hulthén potential given by: 


$$
E_{n l}=-\frac{\delta^{2} \hbar^{2}}{2 \mu}\left\{\frac{\frac{2 \mu Z e^{2}}{\delta \hbar^{2}}-\ell(\ell+1)(1-\omega)}{2(n+\ell+1)}-\frac{n+\ell+1}{2}\right\}^{2}
$$

To confirm the validity of Eq. (35), we have computed the bound state energy eigenvalues as a function of screening parameter $\delta$ for arbitrary values of $\mathrm{n}$ and $\ell$. The data in Table 1 shows the computed energy eigenvalues obtained by using Eq. (35), also shown in Table 1 are corresponding bound state energy eigenvalues of the Hulthén potential in the literature which were obtained numerically (Varshni, 1990) and by asymptotic iteration method (Bayrak et al., 2006). For small values of the screening parameters, our computed bound state energy eigenvalues values are almost indistinguishable from literature results. However, for larger values of the screening parameters such as $\delta=0.035$, for $2 p$ state, our result differs significantly from numerically computed result but in total agreement with those obtained by Bayrak et al. (2006), this trend also applies for the other quantum states, therefore, confirming the applicability of our result for the bound state energy eigenvalues for the Hulthén potential.

Table 1: Energy eigenvalues of the Hulthén potential as a function of screening parameter for $2 p, 3 p, 3 d, 4 p, 4 d$ and $4 f$ states in atomic units $(\hbar=\mu=e=1)$ for $\mathrm{Z}=1$ and $\omega=0.9912122120$

\begin{tabular}{lrrrr}
\hline state & $\delta$ & PR & (Varshi, 1990) & (Bayrak et al., 2006) \\
\hline $2 \mathrm{p}$ & 0.025 & 0.1127603 & 0.1127605 & 0.1128125 \\
& 0.050 & 0.1011512 & 0.1010425 & 0.1012500 \\
& 0.075 & 0.0901725 & 0.0898478 & 0.0903125 \\
& 0.100 & 0.0798243 & 0.0791794 & 0.0800000 \\
& 0.150 & 0.0610195 & 0.0594415 & 0.0612500 \\
& 0.200 & 0.0447368 & 0.0418860 & 0.0450000 \\
& 0.250 & 0.0309760 & 0.0266111 & 0.0312500 \\
& 0.300 & 0.0197372 & 0.0137900 & 0.0200000 \\
$3 \mathrm{p}$ & 0.350 & 0.0110205 & 0.0037931 & 0.0112500 \\
& 0.025 & 0.0437370 & 0.0437069 & 0.0437590 \\
& 0.050 & 0.0333302 & 0.0331645 & 0.0333681 \\
& 0.075 & 0.0243352 & 0.0239397 & 0.0243837 \\
& 0.100 & 0.0167519 & 0.0160537 & 0.0168056 \\
$3 \mathrm{~d}$ & 0.150 & 0.0058206 & 0.0044663 & 0.0058681 \\
& 0.025 & 0.0436937 & 0.0436030 & 0.0437587 \\
& 0.050 & 0.0332546 & 0.0327532 & 0.0333681 \\
& 0.075 & 0.0242384 & 0.0230307 & 0.0243837 \\
& 0.100 & 0.0166448 & 0.0144842 & 0.0168055 \\
$4 \mathrm{p}$ & 0.150 & 0.0057261 & 0.0013966 & 0.0058681 \\
& 0.025 & 0.0199890 & 0.0199625 & 0.0200000 \\
& 0.050 & 0.0112335 & 0.0110582 & 0.0112500 \\
& 0.075 & 0.0049835 & 0.0046219 & 0.0050000 \\
$4 \mathrm{~d}$ & 0.100 & 0.0012390 & 0.0007550 & 0.0012500 \\
& 0.025 & 0.0199671 & 0.0198462 & 0.0200000 \\
& 0.050 & 0.0112006 & 0.0106674 & 0.0112500 \\
$4 \mathrm{f}$ & 0.075 & 0.0049507 & 0.0038345 & 0.0050000 \\
& 0.025 & 0.0199341 & 0.0196911 & 0.0200000 \\
& 0.050 & 0.0111514 & 0.0100620 & 0.0112500 \\
& 0.075 & 0.0049016 & 0.0025563 & 0.0050000 \\
\hline \multirow{4}{*}{. } & & & &
\end{tabular}

\section{Modified Möbius square potential}

If we let $Z=0$ in Eqs. (7) and (26), we have for the modified Möbius square potential:

$$
V_{e f f}(r)=-V_{0}\left(\frac{\mathrm{A}+\mathrm{B} e^{-\delta r}}{1-e^{-\delta r}}\right)^{2}+\frac{L \hbar^{2} \delta^{2}}{2 \mu}\left[\frac{\omega e^{-\delta r}}{1-e^{-\delta r}}+\frac{e^{-2 \delta r}}{\left(1-e^{-\delta r}\right)^{2}}\right]
$$




$$
\begin{aligned}
E_{n l}=-\frac{\delta^{2} \hbar^{2}}{2 \mu}\left\{\frac{\frac{2 \mu V_{0}\left(A^{2}-B^{2}\right)}{\delta^{2} \hbar^{2}}-\ell(\ell+1)(1-\omega)}{2 n+1+\sqrt{(2 \ell+1)^{2}-\frac{8 \mu V_{0}(A+B)^{2}}{\delta^{2} \hbar^{2}}}}-\frac{2 n+1+\sqrt{(2 \ell+1)^{2}-\frac{8 \mu V_{0}(A+B)^{2}}{\delta^{2} \hbar^{2}}}}{4}\right\}^{2}-V_{0} B^{2} \\
-\frac{\delta^{2} \hbar^{2}}{2 \mu}\left\{\frac{2 \mu V_{0}\left(A^{2}-B^{2}\right)}{\delta^{2} \hbar^{2}}-\ell(\ell+1)(1-\omega)\right\}
\end{aligned}
$$

Further, by letting $\delta=2 \alpha$ and $\omega=0$, Eqs. (36) and (37) reduces to the effective potential and bound state energy eigenvalues derived by Okorie et al. (2018)

\section{Deng-Fan potential}

The Deng-Fan potential has been proposed in an attempt to finding a more suitable diatomic potential to describe the vibrational spectrum, qualitatively, it is similar to the Morse potential but has the correct asymptotic behaviour as the internuclear distance approaches zero (Oyewumi et al., 2013). If we choose $A=1, B=-e^{\delta r_{e}}, V_{0}=-D_{e}$ and $Z=0$ , where $r_{e}$ is the equilibrium bond length and $D_{e}$ is the dissociation energy. Eqs. (7) and (26) give respectively for the DengFan potential.

$$
\begin{aligned}
& V_{e f f}(r)=D_{e}\left(1-\frac{e^{\delta r_{e}}-1}{e^{\delta r}-1}\right)^{2}+\frac{L \hbar^{2} \delta^{2}}{2 \mu}\left\{\frac{\omega e^{-\delta r}}{1-e^{-\delta r}}+\frac{e^{-2 \delta r}}{\left(1-e^{-\delta r}\right)^{2}}\right\} \\
& E_{n l}=-\frac{\delta^{2} \hbar^{2}}{2 \mu}\left\{\frac{\frac{2 \mu D_{e}\left(e^{2 \delta r_{e}}-1\right)}{\delta^{2} \hbar^{2}}-\ell(\ell+1)(1-\omega)}{2 n+1+\sqrt{(2 \ell+1)^{2}+\frac{8 \mu D_{e}\left(e^{\delta r_{e}}-1\right)^{2}}{\delta^{2} \hbar^{2}}}}-\frac{2 n+1+\sqrt{(2 \ell+1)^{2}+\frac{8 \mu D_{e}\left(e^{\delta r_{e}}-1\right)^{2}}{\delta^{2} \hbar^{2}}}}{4}\right\}^{2} \\
& +D_{e} e^{2 \delta r_{e}}-\frac{\delta^{2} \hbar^{2}}{2 \mu}\left\{\frac{2 \mu D_{e}\left(e^{2 \delta r_{e}}-1\right)}{\delta^{2} \hbar^{2}}-\ell(\ell+1)(1-\omega)\right\}
\end{aligned}
$$

To test the accuracy of our approximations scheme we have employed equation (39) to compute the energy eigenvalues of various quantum states viz: $2 \mathrm{p}, 3 \mathrm{p}, 3 \mathrm{~d}, 4 \mathrm{p}, 4 \mathrm{~d}, 5 \mathrm{p}, 5 \mathrm{~d}, 5 \mathrm{f}, 6 \mathrm{p}, 6 \mathrm{~d}, 6 \mathrm{f}$ and $6 \mathrm{~g}$ states for the parameter $\omega=0.97685485225$. Tables 2 and 3 , shows the computed energy eigenvalues $(\mathrm{eV})$ in the present $(\mathrm{PR})$ study, also shown in the tables are energy eigenvalues extracted in the literature which were obtained by numerical (NUM) method, Nikiforov-Uvarov(NU) method, Supersymmetric quantum mechanics(SUSY) approach and functional analysis method (FUN). From the results shown in Tables 2 and 3, it is obvious that the energy eigenvalues obtained in the present study is in near perfect agreement with values obtained numerically, thus, confirming the accuracy of the Pekeris-type approximation over the Greene and Aldrich approximation models. 
Table 2: Energy eigenvalues (eV) Ent of the Deng-Fan potential in atomic units $(h=\mu=1)$ with $D_{e}=15$ and $r_{e}=0.40$.

\begin{tabular}{|c|c|c|c|c|c|c|}
\hline state & $\delta$ & PR & NUM & NU & SUSY & FUN \\
\hline \multirow[t]{6}{*}{$2 p$} & 0.05 & 7.86203 & 7.8628 & 7.86080 & 7.86080 & 7.86060 \\
\hline & 0.10 & 7.95542 & 7.95537 & 7.95330 & 7.95330 & 7.95247 \\
\hline & 0.15 & 8.04778 & 8.04724 & 8.04510 & 8.04510 & 8.04322 \\
\hline & 0.20 & 8.13910 & 8.13842 & 8.13620 & 8.13620 & 8.13287 \\
\hline & 0.25 & 8.22942 & 8.22892 & 8.22663 & 8.22663 & 8.22142 \\
\hline & 0.30 & 8.31874 & 8.31874 & 8.31639 & 8.31639 & 8.30889 \\
\hline \multirow[t]{6}{*}{$3 p$} & 0.05 & 10.99840 & 10.9998 & 10.99776 & 10.99780 & 10.99760 \\
\hline & 0.10 & 11.16349 & 11.1647 & 11.16256 & 11.16260 & 11.16170 \\
\hline & 0.15 & 11.32513 & 11.32647 & 11.32425 & 11.32420 & 11.32240 \\
\hline & 0.20 & 11.48333 & 11.48513 & 11.48284 & 11.48280 & 11.47950 \\
\hline & 0.25 & 11.63811 & 11.64068 & 11.63834 & 11.63830 & 11.63310 \\
\hline & 0.30 & 11.78946 & 9.67565 & 11.79076 & 11.79080 & 11.78330 \\
\hline \multirow[t]{6}{*}{$3 d$} & 0.05 & 10.21832 & 10.21651 & 10.21598 & 10.21598 & 10.21540 \\
\hline & 0.10 & 10.35722 & 10.35409 & 10.35354 & 10.35354 & 10.35100 \\
\hline & 0.15 & 10.49337 & 10.48992 & 10.48935 & 10.48935 & 10.48370 \\
\hline & 0.20 & 10.62682 & 10.62403 & 10.62346 & 10.62346 & 10.61350 \\
\hline & 0.25 & 10.75760 & 10.75645 & 10.75591 & 10.75591 & 10.74030 \\
\hline & 0.30 & 10.88575 & 10.88719 & 10.88672 & 10.88672 & 10.86420 \\
\hline \multirow[t]{5}{*}{$4 p$} & 0.05 & 12.49795 & 12.4992 & 12.49760 & 12.49760 & 12.49740 \\
\hline & 0.10 & 12.69715 & 12.69851 & 12.69680 & 12.69680 & 12.69600 \\
\hline & 0.15 & 12.88836 & 12.8901 & 12.88835 & 12.88835 & 12.88650 \\
\hline & 0.20 & 13.07157 & 13.074 & 13.07224 & 13.07224 & 13.06890 \\
\hline & 0.25 & 13.24677 & 13.2501 & 13.24847 & 13.24847 & 13.24330 \\
\hline \multirow[t]{4}{*}{$4 d$} & 0.05 & 12.09957 & 12.0989 & 12.09829 & 12.09829 & 12.09770 \\
\hline & 0.10 & 12.28654 & 12.2857 & 12.28501 & 12.28501 & 12.28250 \\
\hline & 0.15 & 12.46717 & 12.46715 & 12.46642 & 12.46642 & 12.46080 \\
\hline & 0.20 & 12.64152 & 12.64324 & 12.64257 & 12.64257 & 12.63260 \\
\hline \multirow[t]{4}{*}{$4 \mathrm{f}$} & 0.05 & 11.82365 & 11.8209 & 11.82079 & 11.82079 & 11.81950 \\
\hline & 0.10 & 12.00166 & 11.9981 & 11.99796 & 11.99796 & 11.99300 \\
\hline & 0.15 & 12.17420 & 12.1718 & 12.17170 & 12.17170 & 12.16040 \\
\hline & 0.20 & 12.34134 & 12.3421 & 12.34207 & 12.34207 & 12.32210 \\
\hline \multirow[t]{2}{*}{$5 p$} & 0.10 & 13.54218 & 13.5434 & 13.54214 & 13.54214 & 13.54130 \\
\hline & 0.20 & 13.92767 & 13.9301 & 13.92899 & 13.92899 & 13.92570 \\
\hline \multirow[t]{2}{*}{$5 d$} & 0.10 & 13.30717 & 13.3075 & 13.30680 & 13.30680 & 13.30430 \\
\hline & 0.20 & 13.68927 & 13.6931 & 13.69266 & 13.69266 & 13.68270 \\
\hline \multirow[t]{2}{*}{$5 f$} & 0.10 & 13.14872 & 13.1478 & 13.14760 & 13.14760 & 13.14260 \\
\hline & 0.20 & 13.52741 & 13.5333 & 13.53344 & 13.53344 & 13.51340 \\
\hline \multirow[t]{2}{*}{$5 g$} & 0.10 & 13.04026 & 13.0379 & 13.03798 & 13.03798 & 13.02960 \\
\hline & 0.10 & 13.41795 & 13.42667 & 13.42711 & 13.42711 & 13.39380 \\
\hline $6 \mathrm{p}$ & 0.10 & 14.05193 & 14.053 & 14.05209 & 14.05209 & 14.05130 \\
\hline $6 \mathrm{~d}$ & 0.10 & 13.90674 & 13.9075 & 13.90705 & 13.90705 & 13.90450 \\
\hline $6 f$ & 0.10 & 13.81080 & 13.8113 & 13.81119 & 13.81119 & 13.80620 \\
\hline $6 \mathrm{~g}$ & 0.10 & 13.74622 & 13.7466 & 13.74661 & 13.74661 & 13.73830 \\
\hline
\end{tabular}


Table 3: Energy eigenvalues (eV) Ene of the Deng-Fan potential in atomic units $(h=\mu=1)$ with $D_{e}=15$ and $r_{e}=0.80$

\begin{tabular}{|c|c|c|c|c|c|c|}
\hline state & $\delta$ & PR & NUM & NU & SUSY & FUN \\
\hline \multirow[t]{6}{*}{$2 p$} & 0.05 & 4.14177 & 4.14208 & 4.14089 & 4.14089 & 4.14068 \\
\hline & 0.10 & 4.22060 & 4.2204 & 4.21918 & 4.21918 & 4.21835 \\
\hline & 0.15 & 4.29902 & 4.2987 & 4.29739 & 4.29739 & 4.29552 \\
\hline & 0.20 & 4.37703 & 4.3769 & 4.37555 & 4.37555 & 4.37221 \\
\hline & 0.25 & 4.45466 & 4.4551 & 4.45366 & 4.45366 & 4.44845 \\
\hline & 0.30 & 4.53193 & 4.5332 & 4.53175 & 4.53175 & 4.52425 \\
\hline \multirow[t]{6}{*}{$3 p$} & 0.05 & 7.53336 & 7.535 & 7.53279 & 7.53279 & 7.53258 \\
\hline & 0.10 & 7.72556 & 7.7271 & 7.72476 & 7.72476 & 7.72393 \\
\hline & 0.15 & 7.91587 & 7.9177 & 7.91518 & 7.91518 & 7.91330 \\
\hline & 0.20 & 8.10428 & 8.1066 & 8.10404 & 8.10404 & 8.10071 \\
\hline & 0.25 & 8.29081 & 8.2841 & 8.29135 & 8.29135 & 8.28615 \\
\hline & 0.30 & 8.47546 & 8.4799 & 8.47712 & 8.47712 & 8.46962 \\
\hline \multirow[t]{6}{*}{$3 d$} & 0.05 & 5.74196 & 5.7404 & 5.73975 & 5.73975 & 5.73913 \\
\hline & 0.10 & 5.84917 & 5.8465 & 5.84577 & 5.84577 & 5.84327 \\
\hline & 0.15 & 5.95427 & 5.9515 & 5.95068 & 5.95068 & 5.94505 \\
\hline & 0.20 & 6.05732 & 6.0553 & 6.05453 & 6.05453 & 6.04453 \\
\hline & 0.25 & 6.15836 & 6.1582 & 6.15740 & 6.15740 & 6.14177 \\
\hline & 0.30 & 6.25748 & 6.2601 & 6.25932 & 6.25932 & 6.23682 \\
\hline \multirow[t]{5}{*}{$4 p$} & 0.05 & 9.61339 & 9.6156 & 9.61301 & 9.61301 & 9.61280 \\
\hline & 0.10 & 9.88394 & 9.8862 & 9.88352 & 9.88352 & 9.88269 \\
\hline & 0.15 & 10.14866 & 10.1514 & 10.14856 & 10.14856 & 10.14670 \\
\hline & 0.20 & 10.40752 & 10.4111 & 10.40806 & 10.40806 & 10.40470 \\
\hline & 0.25 & 10.66046 & 10.665 & 10.66197 & 10.66197 & 10.65680 \\
\hline \multirow[t]{4}{*}{$4 d$} & 0.05 & 8.49478 & 8.4948 & 8.49334 & 8.49334 & 8.49272 \\
\hline & 0.10 & 8.70898 & 8.7087 & 8.70711 & 8.70711 & 8.70461 \\
\hline & 0.15 & 8.91910 & 8.9194 & 8.91781 & 8.91781 & 8.91218 \\
\hline & 0.20 & 9.12521 & 9.1272 & 9.12551 & 9.12551 & 9.11551 \\
\hline \multirow[t]{4}{*}{$4 \mathrm{f}$} & 0.05 & 7.43817 & 7.4351 & 7.43471 & 7.43471 & 7.43346 \\
\hline & 0.10 & 7.59136 & 7.5868 & 7.58642 & 7.58642 & 7.58142 \\
\hline & 0.15 & 7.74016 & 7.7361 & 7.73573 & 7.73573 & 7.72448 \\
\hline & 0.20 & 7.88468 & 7.8831 & 7.88276 & 7.88276 & 7.86276 \\
\hline \multirow[t]{2}{*}{$5 p$} & 0.10 & 11.30223 & 11.3047 & 11.30207 & 11.30207 & 11.30120 \\
\hline & 0.20 & 11.91217 & 11.9161 & 11.91322 & 11.91322 & 11.90990 \\
\hline \multirow[t]{2}{*}{$5 d$} & 0.10 & 10.52098 & 10.5219 & 10.52009 & 10.52009 & 10.51760 \\
\hline & 0.20 & 11.06710 & 11.0713 & 11.06937 & 11.06937 & 11.05940 \\
\hline \multirow[t]{2}{*}{$5 f$} & 0.10 & 9.79922 & 9.7975 & 9.79666 & 9.79666 & 9.79166 \\
\hline & 0.20 & 10.27014 & 10.2738 & 10.27304 & 10.27304 & 10.25300 \\
\hline \multirow[t]{2}{*}{$5 \mathrm{~g}$} & 0.10 & 9.15766 & 9.1524 & 9.15222 & 9.15222 & 9.14389 \\
\hline & 0.10 & 9.55051 & 9.5528 & 9.55287 & 9.55287 & 9.51954 \\
\hline $6 \mathrm{p}$ & 0.10 & 12.27978 & 12.2822 & 12.27980 & 12.27980 & 12.27900 \\
\hline $6 \mathrm{~d}$ & 0.10 & 11.73667 & 11.7383 & 11.73644 & 11.73644 & 11.73390 \\
\hline $6 \mathrm{f}$ & 0.10 & 11.24582 & 11.2459 & 11.24481 & 11.24481 & 11.23980 \\
\hline $6 \mathrm{~g}$ & 0.10 & 10.81778 & 10.8158 & 10.81533 & 10.81533 & 10.80700 \\
\hline
\end{tabular}

Having established the superiority of the Pekeris-type approximation scheme over the Greene and Aldrich model on the modified Möbius squared potential plus Hulthén potential, we have applied our derived energy eigenvalue equation to a more idealized system, here, we have considered six diatomic molecules: $\mathrm{HCl}, \mathrm{LiH}, \mathrm{TiH}, \mathrm{NiC}, \mathrm{TiC}$ and $\mathrm{ScF}$, the choice of these diatomic molecules is to enable compare our results with those obtained by other authors. The data shown in Table 4 (Oyewumi, Oluwadare, Sen and Babalola, 2012) shows model parameters of the diatomic molecules used in our computation of the bound state energy

Table 4: Spectroscopic parameters of selected molecules, used in the present study.

\begin{tabular}{lrrrr}
\hline molecules & \multicolumn{1}{l}{$\mathrm{De}(\mathrm{eV})$} & \multicolumn{1}{c}{$\mathrm{r}_{\mathrm{e}}(\AA)$} & \multicolumn{1}{c}{$\delta\left(\AA^{-1}\right)$} & \multicolumn{1}{c}{$\mu(\mathrm{amu})$} \\
\hline $\mathrm{HCl}$ & 4.619061175 & 1.2746 & 1.8677 & 0.9801045 \\
$\mathrm{LiH}$ & 2.515283695 & 1.5956 & 1.1280 & 0.8801221 \\
$\mathrm{TiH}$ & 2.05 & 1.781 & 1.32408 & 0.9873710 \\
$\mathrm{NiC}$ & 2.76 & 1.621 & 2.25297 & 9.974265 \\
$\mathrm{TiC}$ & 2.66 & 1.790 & 1.52550 & 9.606790 \\
$\mathrm{ScF}$ & 5.85 & 1.794 & 1.46102 & 13.358942 \\
\hline
\end{tabular}

The results shown in Tables 5 and 6 are the computed bound state energy eigenvalues for $\mathrm{HCl}, \mathrm{LiH}, \mathrm{TiH}, \mathrm{NiC}, \mathrm{TiC}$ and $\mathrm{ScF}$. We have included columns for bound state energy eigenvalues of the Deng-Fan potential computed within the frameworks of 
Nikiforov-Uvarov (NU) method, comparison of the present results (PR) and that obtained by NU method shows good agreement for the state between our computed energy eigenvalues and those obtained by NU method, however, there is no available data on results obtained by numerical methods to enable further comparison. The plots in Figures 1 and 2 are the radial wave functions for $2 \mathrm{p}, 5 \mathrm{f}$ for $\mathrm{HCl}$ and $1 \mathrm{~s}, 4 \mathrm{f}$ for $\mathrm{LiH}$.

Table 5: The bound state energies $E_{n l}$ (in $\mathrm{eV}$ ) for $\mathrm{HCl}, \mathrm{LiH}$ and $\mathrm{TiH}$

\begin{tabular}{llllllll}
\hline state & \multicolumn{1}{l}{ HCl } & \multicolumn{2}{l}{ LiH } & \multicolumn{3}{l}{ TiH } & N \\
& & PR & NR & NU & PR & NU \\
\hline $\mathbf{0}$ & 0 & 0.202058236 & 0.201984174 & 0.103333625 & 0.103334650 & 0.095194192 & 0.095195135 \\
& 1 & 0.204074322 & 0.204854248 & 0.104898886 & 0.105236729 & 0.096217575 & 0.096647888 \\
$\mathbf{1}$ & 0 & 0.590952203 & 0.590747827 & 0.302003040 & 0.302005955 & 0.277946427 & 0.277949104 \\
& 1 & 0.592885903 & 0.593537612 & 0.303497609 & 0.303838653 & 0.278925122 & 0.279358078 \\
$\mathbf{2}$ & 0 & 0.960325653 & 0.960011044 & 0.490681252 & 0.490685861 & 0.450920200 & 0.450924416 \\
& 1 & 0.962178048 & 0.962721591 & 0.492106738 & 0.492450759 & 0.451854842 & 0.452290234 \\
& 2 & 0.965881747 & 0.968141645 & 0.494956099 & 0.495978997 & 0.453723485 & 0.455021255 \\
$\mathbf{3}$ & 0 & 1.310433982 & 1.310027865 & 0.669594907 & 0.669601019 & 0.614253922 & 0.614259487 \\
& 1 & 1.312206130 & 1.312660203 & 0.670952866 & 0.671299648 & 0.615145130 & 0.615582758 \\
& 2 & 1.315749352 & 1.317923855 & 0.673667213 & 0.674695388 & 0.616926918 & 0.618228694 \\
& 3 & 1.321061502 & 1.325816775 & 0.677734812 & 0.679785205 & 0.619598028 & 0.622196086 \\
$\mathbf{4}$ & 0 & 1.641527447 & 1.641047243 & 0.838963132 & 0.838970564 & 0.768082994 & 0.768089723 \\
& 1 & 1.643220385 & 1.643602379 & 0.840255067 & 0.840604402 & 0.768931376 & 0.769371041 \\
& 2 & 1.646605204 & 1.648711644 & 0.842837407 & 0.843870601 & 0.770627521 & 0.771933083 \\
& 3 & 1.651679792 & 1.656373023 & 0.846707095 & 0.848766203 & 0.773170195 & 0.775774658 \\
& 4 & 1.658440980 & 1.666583499 & 0.851859551 & 0.855286782 & 0.776557543 & 0.780893985 \\
$\mathbf{5}$ & 0 & 1.953851289 & 1.953313156 & 0.998997828 & 0.999006401 & 0.912539887 & 0.912547598 \\
& 1 & 1.955466034 & 1.955792078 & 1.000225194 & 1.000576880 & 0.913346036 & 0.913787546 \\
& 2 & 1.958694483 & 1.960748932 & 1.002678435 & 1.003716397 & 0.914957727 & 0.916266858 \\
& 3 & 1.963534556 & 1.968181734 & 1.006354573 & 1.008422072 & 0.917373744 & 0.919984364 \\
& 4 & 1.969983136 & 1.978087513 & 1.011249142 & 1.014689589 & 0.920592266 & 0.924938312 \\
& 5 & 1.978036066 & 1.990462308 & 1.017356201 & 1.022513206 & 0.924610865 & 0.931126367 \\
\hline
\end{tabular}

Table 6: The bound state energies $E_{n l}$ (in $\mathrm{eV}$ ) for $\mathrm{NiC}$, TiC and $\mathrm{ScF}$

\begin{tabular}{|c|c|c|c|c|c|c|c|}
\hline \multirow{2}{*}{\multicolumn{2}{|c|}{ state }} & \multicolumn{2}{|l|}{$\mathrm{NiC}$} & \multicolumn{2}{|l|}{$\mathrm{TiC}$} & \multicolumn{2}{|l|}{$\mathrm{ScF}$} \\
\hline & & PR & NU & PR & NU & PR & NU \\
\hline \multirow[t]{2}{*}{$\mathbf{0}$} & 0 & 0.055343810 & 0.055344362 & 0.039112902 & 0.039113293 & 0.047569741 & 0.047570217 \\
\hline & 1 & 0.055451873 & 0.055579192 & 0.039212882 & 0.039272617 & 0.047642645 & 0.047682126 \\
\hline \multirow[t]{2}{*}{1} & 0 & 0.164292441 & 0.164294062 & 0.116392271 & 0.116393424 & 0.142067832 & 0.142069247 \\
\hline & 1 & 0.164399275 & 0.164527691 & 0.116491037 & 0.116551564 & 0.142140230 & 0.142180660 \\
\hline \multirow[t]{3}{*}{2} & 0 & 0.270944879 & 0.270947523 & 0.192438048 & 0.192439939 & 0.235730886 & 0.235733223 \\
\hline & 1 & 0.271050486 & 0.271179952 & 0.192535604 & 0.192596892 & 0.235802779 & 0.235844142 \\
\hline & 2 & 0.271261700 & 0.271644807 & 0.192730711 & 0.192910789 & 0.235946566 & 0.236065981 \\
\hline \multirow[t]{4}{*}{3} & 0 & 0.375304805 & 0.375308427 & 0.267253990 & 0.267256594 & 0.328560483 & 0.328563726 \\
\hline & 1 & 0.375409188 & 0.375539658 & 0.267350339 & 0.267412364 & 0.328631873 & 0.328674152 \\
\hline & 2 & 0.375617952 & 0.376002118 & 0.267543033 & 0.267723901 & 0.328774651 & 0.328895002 \\
\hline & 3 & 0.375931094 & 0.376695804 & 0.267832066 & 0.268191197 & 0.328988816 & 0.329226276 \\
\hline \multirow[t]{5}{*}{4} & 0 & 0.477375891 & 0.477380444 & 0.340843838 & 0.340847133 & 0.420558199 & 0.420562331 \\
\hline & 1 & 0.477479052 & 0.477610482 & 0.340938984 & 0.341001723 & 0.420629086 & 0.420672264 \\
\hline & 2 & 0.477685371 & 0.478070549 & 0.341129272 & 0.341310904 & 0.420770858 & 0.420892131 \\
\hline & 3 & 0.477994846 & 0.478760649 & 0.341414695 & 0.341774668 & 0.420983514 & 0.421221926 \\
\hline & 4 & 0.478407469 & 0.479680772 & 0.341795242 & 0.342393002 & 0.421267051 & 0.421661649 \\
\hline \multirow[t]{6}{*}{5} & 0 & 0.577161800 & 0.577167239 & 0.413211313 & 0.413215272 & 0.511725603 & 0.511730608 \\
\hline & 1 & 0.577263740 & 0.577396081 & 0.413305260 & 0.413368688 & 0.511795988 & 0.511840049 \\
\hline & 2 & 0.577467619 & 0.577853763 & 0.413493149 & 0.413675522 & 0.511936756 & 0.512058932 \\
\hline & 3 & 0.577773432 & 0.578540282 & 0.413774975 & 0.414135763 & 0.512147906 & 0.512387253 \\
\hline & 4 & 0.578181173 & 0.579455631 & 0.414150724 & 0.414749401 & 0.512429435 & 0.512825013 \\
\hline & 5 & 0.578690835 & 1.022513206 & 0.414620383 & 0.931126367 & 0.512781339 & 1.022513206 \\
\hline
\end{tabular}




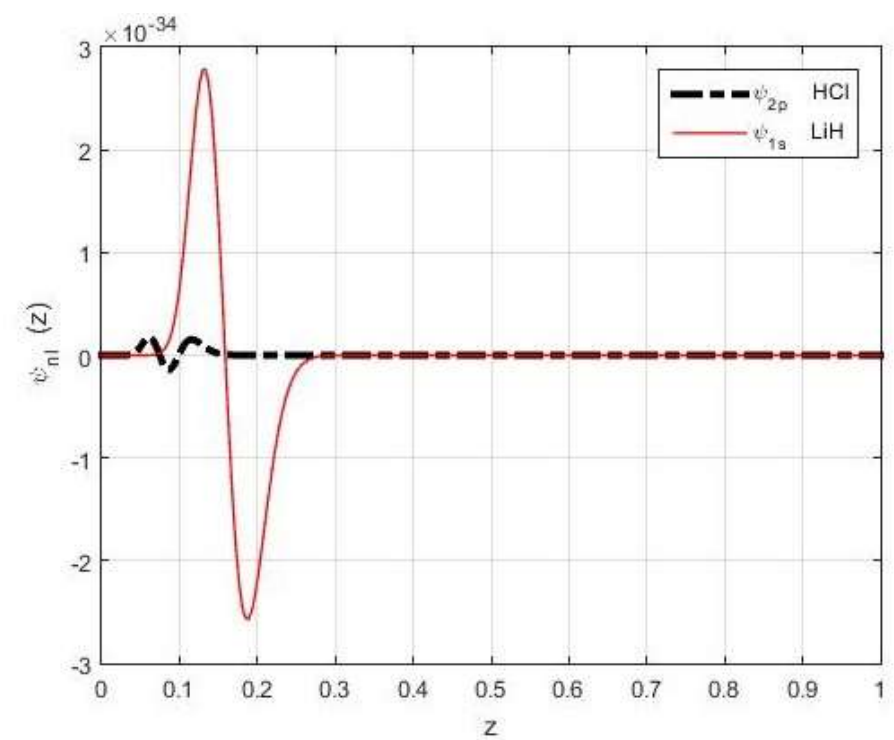

Figure 1: Plot of unnormalised radial wave functions of $\mathrm{HCl}$ and $\mathrm{LiH}$ for $2 \mathrm{p}$ and 1 s states

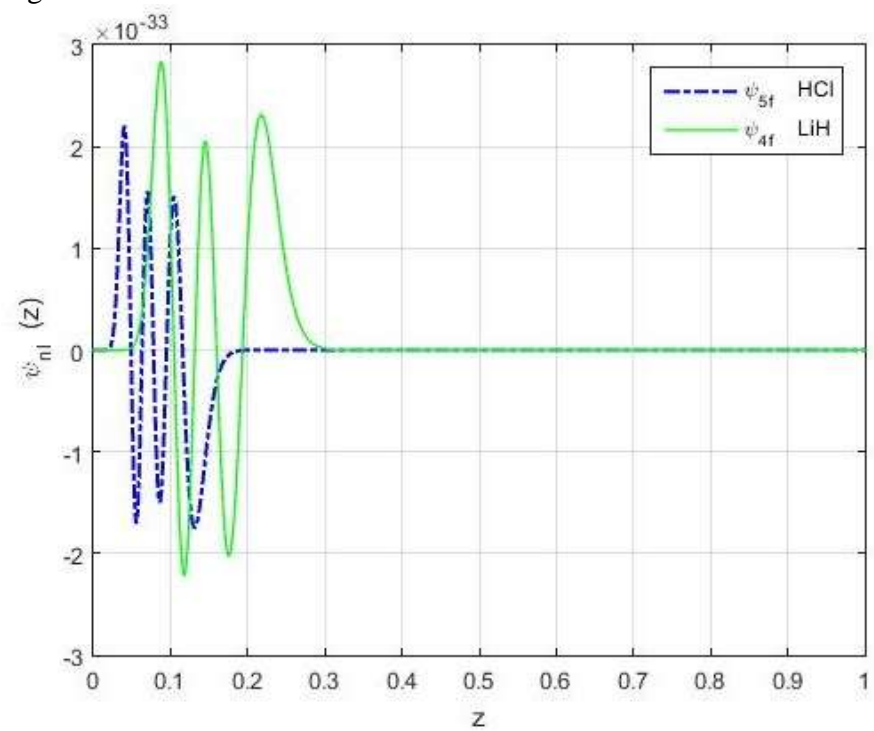

Figure 2: Plot of unnormalised radial wave functions of $\mathrm{HCl}$ and $\mathrm{LiH}$ for $5 \mathrm{f}$ and $4 \mathrm{f}$ states

\section{CONCLUSION}

We have solved for the approximate $\ell$-state solution of time independent Schrödinger wave equation with modified Möbius squared potential plus Hulthén potential, the Pekeristype approximation scheme was used to simplify the spinorbit centrifugal term. we have obtained closed form expressions for the bound state energy eigenvalues and normalized radial wave functions of the modified Möbius squared potential plus Hulthén potential, using our results we have derived expressions for the bound state energy eigenvalues and radial wave functions of Hulthén potential, modified Möbius squared potential and Deng-Fan potential, by appropriately selecting the parameter $\omega$, we have computed bound state energy eigenvalues for Hulthén potential and Deng-fan potential for arbitrary values of quantum numbers, we have also obtained the bound state energy eigenvalues for the Deng-fan potential for six diatomic molecules: $\mathrm{HCl}, \mathrm{LiH}, \mathrm{TiH}, \mathrm{NiC}, \mathrm{TiC}$ and $\mathrm{ScF}$, our computed results compared favorably with existing results in the literature. The results obtained in this research work can find useful applications in the area of solid state physics, atomic physics, molecular physics and chemical physics

\section{REFERENCES}

Awoga O.A. and Ikot A.N. (2012). Approximate solution of Schrödinger equation In the D- dimensions for inverted generalized hyperbolic potential. Indian Academy of Science. Pramana Journal of Science 79, (345-356)

Bayrak. O, Kocak. G and Boztosun .I (2006). Any $\ell$ state solution of the Hulthén potential by The asymptotic iteration method. Journal of Physics: mathematical and General 39 11521-11529

Chen Z-Y.,Li M. and Jia C-H. (2009).Approximate analytical solutions of the Schrödinger equation with the ManningRosen potential model. Modern Physics Letters A. 24, (18631874)

Dong S-H and Gu X-Y (2007). Arbitrary $\ell$ - state solutions of the Schrödinger equation with the Deng-fan molecular 
potential. Journal of physics conference series 96 doi0.1088/1742-6596/96/1/012109

Edet C. O, Okorie, U. S, Ngiangia, A.T. and Ikot, A.N. (2019). Bound state solutions of the Schrodinger equation for the modified Kratzer plus screened coulomb potential. Indian journal of Physics $\mathbf{9 4}$ 425-433

Eyube E.S., Sanda A. and Y.Y. Jabil (2019). $\ell$ - Wave Analytical Solutions of Schrödinger Equation with Tietz-Hua Potential. Journal of the Nigerian Association of Mathematical Physics, 52, (223-230)

Hitler, L., Ita, B.I., Akakuru, O.U., Magu, T, O., Joseph, I. and Pigweh, A. (2017). Radial Solution of the s-Wave Schrödinger Equation with Kratzer plus Modified Deng-Fan Potential under the Framework of Nikiforov-Uvarov Method. International Journal of Applied Mathematics and Theoretical Physics, 3, (97-100)

Ikdair S. M (2009). An improved approximation scheme for the centrifugal term and Hulthén potential. The European Physical Journal A 39, (307-314)

Ikot A.N., Awoga O.A., Hassanabadi H and Maghsoodi Elham (2014). Analytical approximate Solutions of Schrödinger Equation in $D$ Dimensions with Exponential Type Potentials For arbitrary states Journal of theoretical Physics. 61, (457-463)

Ita B.I., Nzeata-Ibe N., Magu T.O. and Hitler L. (2018), Bound State Solutions of the Schrödinger Equation with Woods-Saxon plus Inversely Quadratic Potential via Parametric Nikiforov-Uvarov Method. Manila Journal of Science. 11, (58-67)

Jia C-S, Liu J-Y and Wang P-Q (2008). A new approximation scheme for the centrifugal term and the Hulthén potential. Physics letters A (4779-4782).

Meyur.S and Debnath. S (2009). Solutions of the Schrödinger Equation with Hulthén plus Manning- Rosen Potential Latin American Journal of Physics Edu. 3.(300-306)

Miranda M.G., Sun G-U. and Dong S-H. (2010). The Solution of the Second Pöschl-Teller Like Potential by NikiforovUvarov Method. International Journal of Modern Physics E 19, (123-129)

Nyengeri H., Simbizi R., Girukwishaka A., Nizigiyimana R and Ndenzako E. (2018).Fröbenius Series Solutions of the Schrödinger Equation with Various Type of Symmetric Hyperbolic Potentials in one Dimension. Open access journal 1104728 Doi:10.4236/oalib.1104728

Okorie U.S., Ikot A.N., Onyeaju M.C. and Chukwuocha E.O. (2018). Bound State Solutions of the Schrödinger Equation with Modified Mobius Square Potential and its Thermodynamic Properties. Journal of Molecular Modelling. 24, 289
Oyewumi k.J., Oluwadare.O.J., Sen K.D and Babalola O.A. (2013). Bound state solutions of the Deng-fang potential with Pekeris type approximation using, Nikiforov-Uvarov Method Journal of math and chemistry 51,976-991

Pahlavani. R M.,Rahbar .H and Ghezelbash M.(2013). Relativistic Schrödinger Wave

Equation for Hydrogen Atom Using Factorization Method. Journal of

Microphysics 3 (1-7)

Qiang W-C., Gao. Y and Zhou R-S. (2007). Arbitrary $\ell$-State Approximate of the Hulthén Potential through the Exact quantization rule. Central European Journal of Physics Letters. 6,2, (356-362)

Qiang W-C., Li K. and Chen W-L. (2009). New Bound and Scattering State Solutions of the Manning-Rosen Potential with the Centrifugal Term. J. Phys. A: Math. Theor. 42 (2009) 205306 (13pp)

Rahbar,H and Sadeghi J.(2016).Solving the Schrödinger equation with Hartmann potential

by factorization method and supersymmetry. Theoretical physics 1, (07-13)

Saxena A.K (2012). Textbook of quantum mechanics, second edition C B S publishers' pp 150

Taskin, F.and Kocak. G (2010). Approximate solution of Schrödinger equation for Eckart Potential with the centrifugal term. Chinese Journal of Physics B 19 (3141-3146)

Tsaur G-Y and Wang J. (2014). A Universal LaplaceTransform Approach to Solving Schrödinger Equation for all Known Solvable Models. Eur. J. Phys. 35015006 (17pp)

Varshni. Y P (1990). Eigenenergies and oscillator strengths for the Hulthén potential Phys. Rev A. 41, 4682

Wei G-F. and Dong S-H. (2008). Approximately Analytical Solutions of the Manning-Rosen Potential with the Spin-Orbit Coupling Term and Spin Symmetry. Physics Letters A. 373, (49-53)

Wei G-F. and Dong S-H. (2010). Pseudospin Symmetry in the relativistic Manning-Rosen Potential including a Pekeris-type approximation to the pseudo-centrifugal term. Physics Letters B. 686, (288-292)

Yazarloo B.H., Hassanabadi .H and Zarrinkama. S (2012). Oscillator strengths based Möbius square potential under Schrödinger Equation. The European Journal ofPhysics 127:51

Zhang LH,Li XP,Jia CS (2011)Approximate solutions of the Schrödinger equation with the generalized Morse potential model including the centrifugal term. International Journal of Quantum Chemistry 111,9(870-1878) 